\title{
Participation of Women in Engineering \& Technology Education and Employment
}

\author{
Addissie Melak, Seema Singh
}

\begin{abstract}
Engineering and Technology Education is a means to sustain and accelerate the overall development in a country and it has a direct effect on individuals' productivity and earnings as well. But the problem is there is gender disparity in distribution especially in developing countries among educational branches. In Ethiopia, despite significant improvements in the last couple of years, women are still under-represented in engineering and technology. Since the share of Ethiopian women in science and technology programs in higher education has been low, the governments apply a policy to stream $70 \%$ of all university entrants to be in the science and technology track indirectly to increase their entry into these fields. Even if this types of policy is applying, their participation in engineering and technology education and employment is very low proportion. The main purpose of this study is an attempt to assess trends and share of women in the field of engineering and technology regarding to their share of enrollment, employment and professional positions from the period $2000-12$ based on available secondary data collected from different sources. The collected quantitative data were analyzed by using descriptive data analysis techniques. Result from the data shows that there is low share of women in engineering and technology enrollment, employment and professional position status as compared to male in the country. Even if the share of enrollment of women in higher education is on improved status, their share of enrollment in engineering and technology filed is quite low that on average below 30 percent per year. In addition, this grate disparity and low participation of women in engineering and technology invites further investigation regarding to what is the reason behind.

Key words: Women, engineering and technology, enrollment and employment, Ethiopia
\end{abstract}

\section{INTRODUCTION}

Ethiopia is found in eastern part of Africa. The total population accounts 102.4 million with life expectancy of 65 years and GNI per capita is \$1750 in 2016 [11], [29] and women accounts half of the population.Since education is a means to sustain and accelerate the overall development in a country and it has a direct effect on individuals' productivity and earnings as well [30], the Ethiopian government has adopted a new Education and Training Policy in 1994. The policy focuses on increasing access to educational opportunities with enhanced equity, quality and relevance

Revised Manuscript Received on March 13, 2020.

* Correspondence Author

Addissie Melak*, Department of Humanities Delhi Technological University, Delhi, India,

Department of Economics, Debre Tabor University, Ethiopia. E-mail addmelak24@gmail.com

Professor Seema Singh, Professor, Department of Humanities, Delhi Technological University, Delhi, India. E-mailseemahumanitiesdtu@gmail.com

(c) The Authors. Published by Blue Eyes Intelligence Engineering and Sciences Publication (BEIESP). This is an open access article under the CC BY-NC-ND license (http://creativecommons.org/licenses/by-nc-nd/4.0/) stared in 1997/98 with the long-term goal of achieving universal primary education [31] and is governed by Ministry of education. Ethiopia school enrolment in 2015/ 2016 at the age $7-18$ is male at primary level $64.3 \%$ and at secondary level $5.5 \%$ as well as female at primary level is $64.2 \%$ and secondary level $5.8 \%$. Tertiary education enrolment rate in 2014 of male is $10.94 \%$ and $5.28 \%$ female [32]. Total literacy rate in the same year was $64.1 \%[12]$.

Women were totally excluded from education life for long time history of Ethiopia. There were strong social and cultural pressures for girls to marry before 14 years old. Virginity before marriage was highly valued and unmarried girl over 14 year was stigmatized. Girls are socialized to be shy and obedient and not to speak in front of adults, particularly in front of men. The focus is on her future role as respectful wife and good mother, hence they didn't went to school, even they went because of various problem such as harassment , violence [1] and their own natural phenomena of adolescence age , they dropout from school. This situation again leads to low employment participation in countries development. Hence to encourage women in education and employment participation, Ethiopian government policy has supported affirmative action for women since its beginning in 1994. Since the time women started to come out of domestic chores in Ethiopia, they have made significant contribution in all spheres of activities vital to the country development [27]-[28].

However, still, women are underrepresented in engineering and technology education and employment [3], [9], [5], [17], [18]. There are various factors for this under representation. Such as studies by [2], [8], [21], [14] [13], [25] found high school educational background, family education and lack of role models have an impact on decision to study engineering and technology. Studies [16], [19], [17], [24]; [6], [22], [23], [26]found that employment participation of women engineering and technology is low and is male dominated sector. However, there are few study about participation status of women in engineering and technology in Ethiopia. This indicates that there is research gap in the study area which need more research investigation. Thus, the objective of this study is to examine the trends and share of women in engineering and technology education and employment participation with the help of secondary data.

\section{METHODOLOGY OF THE STUDY}

In this study secondary data from Ethiopia Ministry of Education statistics annual abstract report and National Assessment [10], [28] were taken to analyze the trends and share of women participation in engineering and technology education and employment. Result analysis of this study has done through the following procedure.

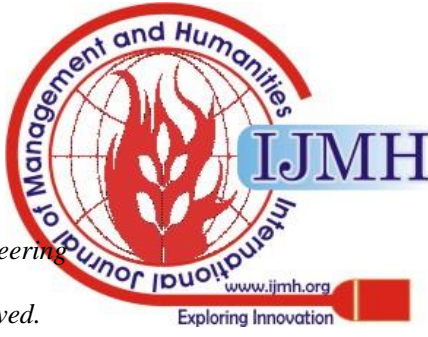




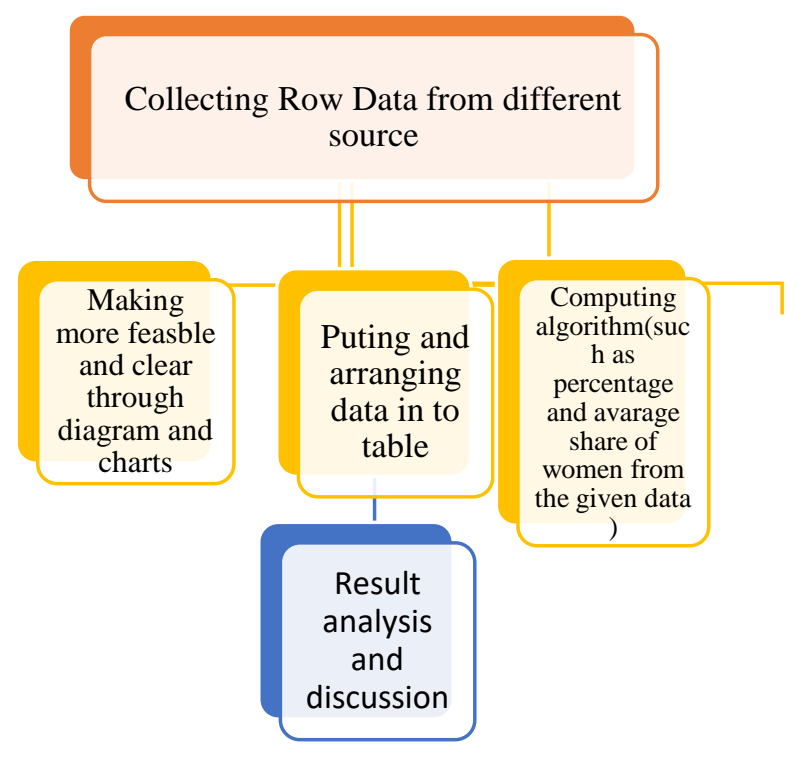

\section{RESULTS AND ANALYSIS}

Ethiopian Education system is structured by different stages which is kindergarten, alternative basic education, $1^{\text {st }}$ cycle primary education (grade $1-4), 2^{\text {nd }}$ cycle primary level from grade 5-8, $1^{\text {st }}$ cycle secondary level grade 9 and $10,2^{\text {nd }}$ cycle secondary education level (preparatory school grade 11 and12), Technical and vocational education and training (TVET) , Undergraduate degree , Master degree and Ph.D education level. Hence, in this study researchers going to investigate about participation of women in engineering and technology education and employment from preparatory school (grade 11 and 12) share of enrollment since this education level is the base for joining to higher education institutions.

Table-I: Trends of Women Enrollment in Preparatory School Program

\begin{tabular}{|c|c|c|c|c|}
\hline \multirow[t]{2}{*}{ Year } & \multicolumn{4}{|c|}{ Enrollment } \\
\hline & Boys & Girls & Total & \% girls \\
\hline $\begin{array}{l}\text { 1995E.C } \\
(2002 / 03)\end{array}$ & 56367 & 22274 & 78641 & $28.32 \%$ \\
\hline $\begin{array}{l}\text { 1996E.C } \\
(2003 / 04)\end{array}$ & 68,330 & 25,065 & 93,395 & $26.83 \%$ \\
\hline $\begin{array}{l}1997 \text { E.C } \\
(2004 / 05)\end{array}$ & 67,413 & 25,070 & 92,483 & $27.1 \%$ \\
\hline $\begin{array}{l}1998 \text { E.C } \\
(2005 / 06)\end{array}$ & 91,889 & 31,794 & 123,683 & $25.7 \%$ \\
\hline $\begin{array}{l}1999 \text { E.C } \\
(2006 / 07)\end{array}$ & 117,000 & 58,219 & 175,219 & $33.23 \%$ \\
\hline $\begin{array}{l}\text { 2000E.C } \\
(2007 / 08)\end{array}$ & 130,533 & 62,911 & 193,444 & $32.52 \%$ \\
\hline $\begin{array}{l}\text { 2001E.C } \\
(2008 / 09)\end{array}$ & 146,547 & 58,713 & 205,260 & $28.6 \%$ \\
\hline $\begin{array}{l}\text { 2002E.C } \\
(2009 / 10)\end{array}$ & 156194 & 86886 & 204,308 & $42.5 \%$ \\
\hline $\begin{array}{l}\text { 2003E.C } \\
\text { (2010/11 }\end{array}$ & 169,571 & 118,645 & 288,216 & $\begin{array}{l}41.165 \\
\%\end{array}$ \\
\hline $\begin{array}{l}\text { 2004E.C } \\
(2011 / 12)\end{array}$ & 172,268 & 156,724 & 328,992 & $47.64 \%$ \\
\hline Average & & & & $33.36 \%$ \\
\hline
\end{tabular}

Source : [28]
The trends of share of female in preparatory school (grade 11 and 12) given in table-I, shows experience of improvement with fluctuation through time. The percentage of female students during 2011/12 has sharply increased and has reached $47 \%$ but when we observe the average percentage share of female $33.36 \%$ which show the there is some existence of gap.

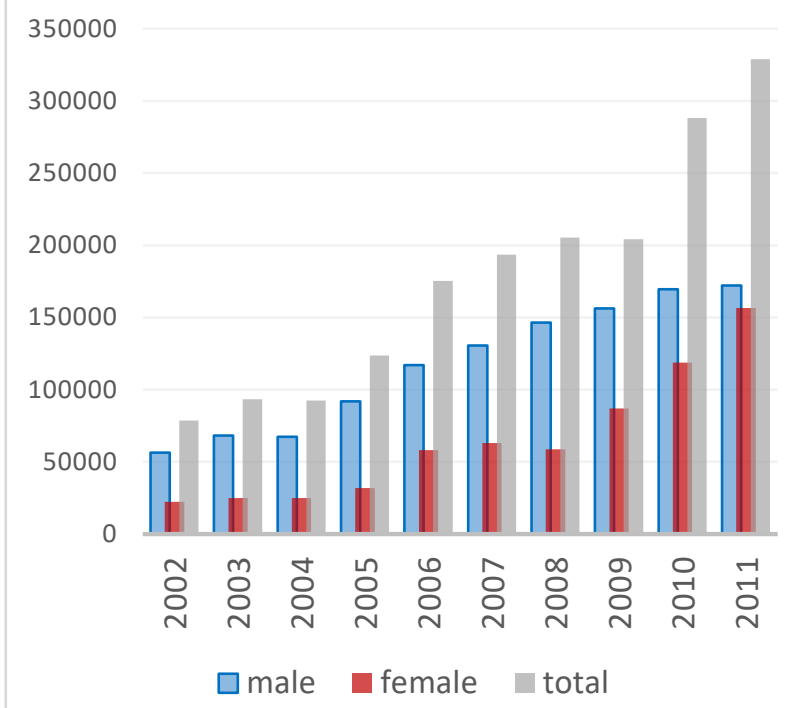

Fig. 1. Women students' enrollment in preparatory school program

Figure 1 clearly show, the share of female students in in preparatory school (grade 11 and 12) improved in the last ten years. This is due to some affirmative action taken for girls able to join in preparatory school.

From table-II, the trends of share of women in higher education is very low in the last 10 years which is an average of $24.98 \%$. This is due to various factors social, cultural, economic and personal factors which is beyond the scope of this study which needs further investigation. However, there was a better improvement of share of women during the year 2001, 2008, and 2011 which is around 28\%.

Table-II: Trends of women and Man Enrollment in Ethiopian Universities

\begin{tabular}{|l|l|l|l|l|}
\hline Year & \multicolumn{4}{|c|}{ Enrolment } \\
\hline \multicolumn{1}{|c|}{ E.C } & Man & Women & Total & \%Women \\
\hline $\begin{array}{l}\text { 1993 } \\
(2000 / 01)\end{array}$ & 9594 & 45526 & $21.07 \%$ \\
\hline $\begin{array}{l}\text { 1994E.C } \\
(2001 / 02)\end{array}$ & 32279 & 13517 & 46796 & $28.88 \%$ \\
\hline $\begin{array}{l}\text { 1995E.C } \\
(2002 / 03)\end{array}$ & 57321 & 18676 & 75997 & $24.57 \%$ \\
\hline $\begin{array}{l}\text { 1996E.C } \\
(2003 / 04)\end{array}$ & 59352 & 18375 & 77727 & $23.64 \%$ \\
\hline $\begin{array}{l}\text { 1997E.C } \\
(2004 / 05)\end{array}$ & 70388 & 21267 & 91655 & $23.2 \%$ \\
\hline $\begin{array}{l}\text { 1998E.C } \\
(2005 / 06)\end{array}$ & 70388 & 21267 & 91655 & $23.2 \%$ \\
\hline $\begin{array}{l}\text { 1999E.C } \\
(2006 / 07)\end{array}$ & 79465 & 25108 & 104573 & $24 \%$ \\
\hline $\begin{array}{l}\text { 2000E.C } \\
(2007 / 08)\end{array}$ & 206336 & 64020 & 270356 & $23.67 \%$ \\
\hline
\end{tabular}

\section{Published By:}

Blue Eyes Intelligence Engineeringes

\& Sciences Publication

(C) Copyright: All rights reserved.

and $\mathrm{Hu}_{\mathrm{H}}$

Copyright: All rights reserved. 


\begin{tabular}{|l|l|l|l|l|}
\hline $\begin{array}{l}\text { 2001E.C } \\
(2008 / 09)\end{array}$ & 229489 & 91338 & 320827 & $28.46 \%$ \\
\hline $\begin{array}{l}\text { 2002E.C } \\
(2009 / 10)\end{array}$ & 319327 & 115332 & 434659 & $26.5 \%$ \\
\hline $\begin{array}{l}2003 E . C \\
(2010 / 11)\end{array}$ & 344137 & 123706 & 467843 & $26.44 \%$ \\
\hline $\begin{array}{l}2004 \mathrm{E} . C \\
(2011 / 12\end{array}$ & 353163 & 138708 & 491871 & $28.2 \%$ \\
\hline $\begin{array}{l}\text { 2005E.C } \\
(2012 / 13)\end{array}$ & 553848 & 166141 & 719989 & $23 \%$ \\
\hline
\end{tabular}

Figure 2 which is constructed [28] from table-II shows, the enrollment status of trends of women in in Ethiopian higher education which indicates there is high gender disparity in enrollment in this level of education.

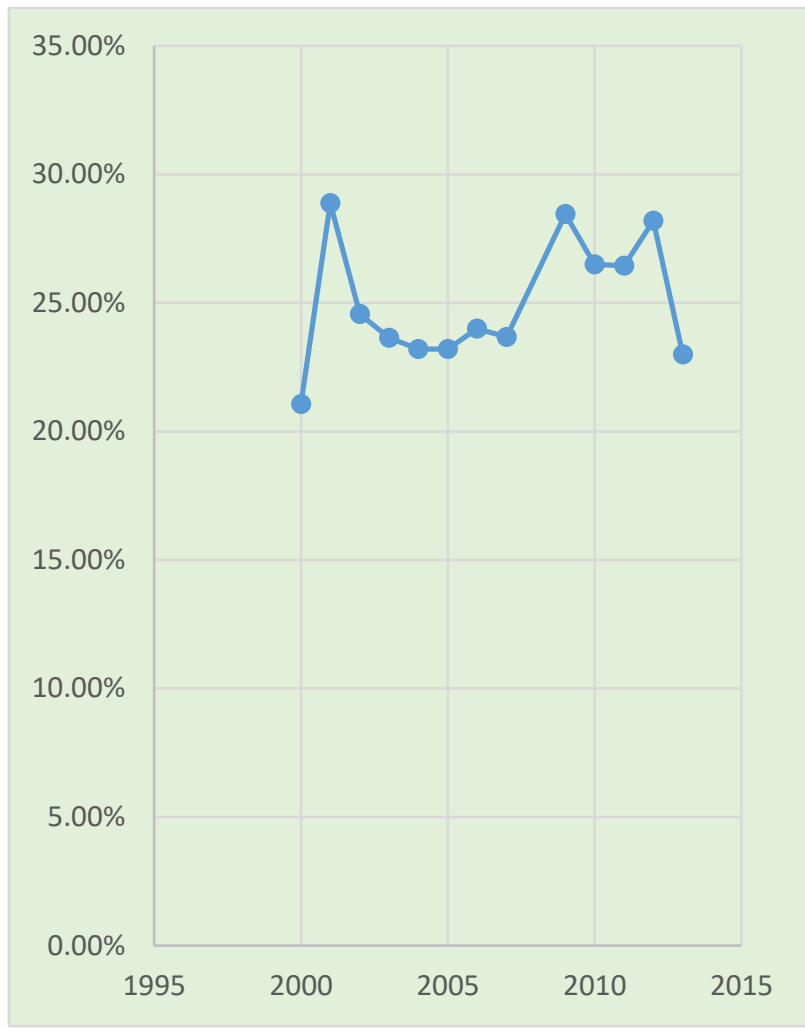

Fig. 3. Percentage of women enrollment in higher

education from the total. Source: [28]

Fig. 2. Share of women' enrollment in Ethiopian Universities

Table-III: Trends of Women in Higher Education Enrolment, and Graduation in Undergraduate and Post Graduate Program

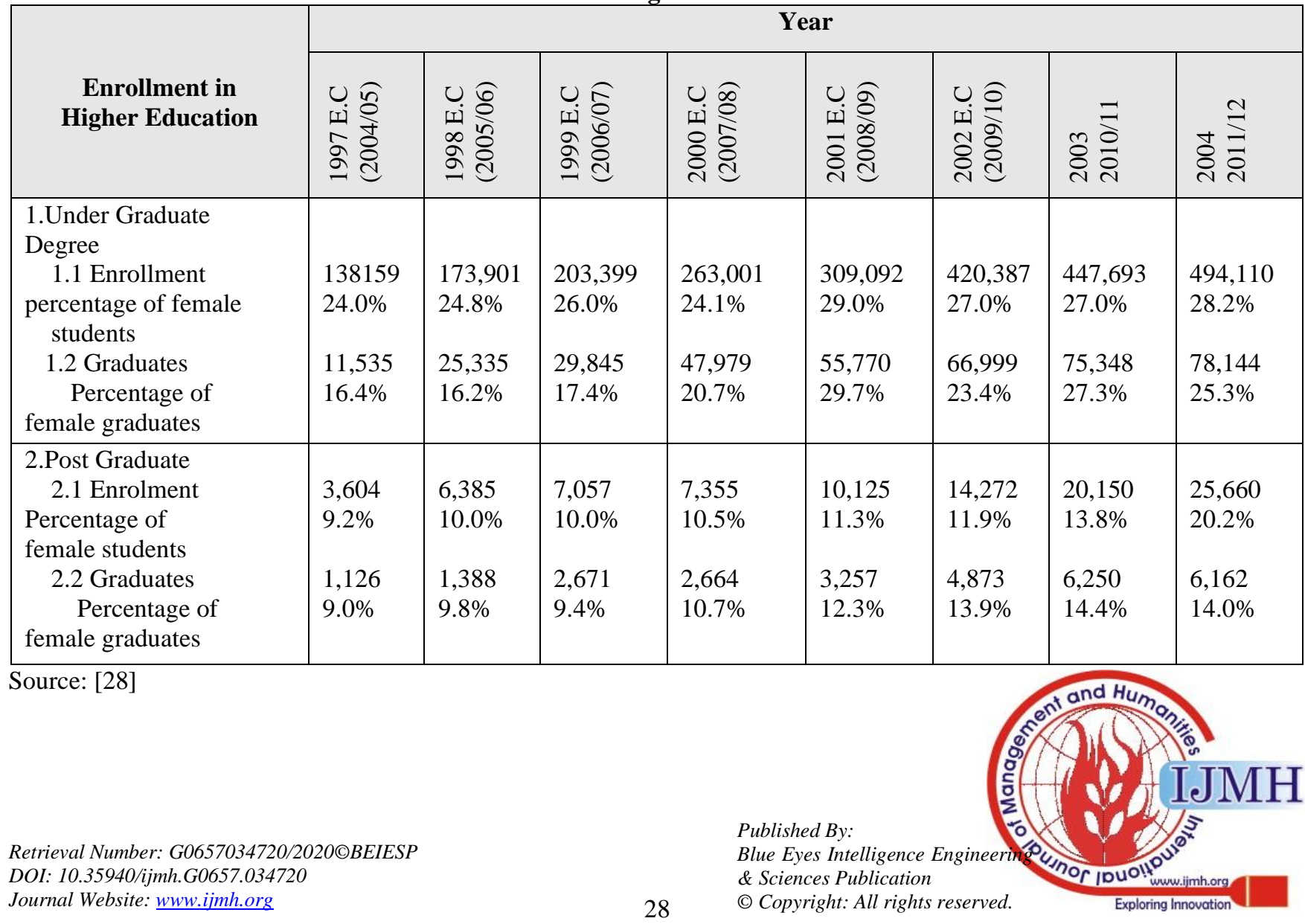


In figure 3, the percentage share of women enrolment from the total enrollment of higher education had shown increasing trend and falling in 2002, which constantly growing till 2008.

Again in table-III, trends of share of women in higher education enrolment, and graduation in undergraduate and post graduate program. From this the share of enrollment in undergraduate program is not that much improved but the share of graduation in under graduate program shows some improvement trend. Their share of enrollment in post graduate level is increased from $9.2 \%$ to $20.2 \%$ in the last 8 consecutive years.

Table-IV: Trends of Share of Women Enrollment and Graduation in Undergraduate Program

\begin{tabular}{|l|l|l|l|l|}
\hline \multirow{2}{*}{ Year } & \multicolumn{2}{l|}{$\begin{array}{l}\text { Under graduate } \\
\text { Enrollment }\end{array}$} & \multicolumn{2}{l|}{$\begin{array}{l}\text { Undergraduate } \\
\text { Graduates }\end{array}$} \\
\cline { 2 - 5 } & Female & Total & Female & Total \\
\hline 2004 & 33158 & 138,159 & 1892 & 11,535 \\
\hline 2005 & 43127 & 173,901 & 4104 & 25,335 \\
\hline 2006 & 52884 & 203,399 & 5193 & 29,845 \\
\hline 2007 & 63383 & 263,001 & 9932 & 47,979 \\
\hline 2008 & 89637 & 309,092 & 16563 & 55,770 \\
\hline 2009 & 113505 & 420,387 & 15678 & 66,999 \\
\hline 2010 & 120877 & 447,693 & 20570 & 75,348 \\
\hline 2011 & 139339 & 494,110 & 19770 & 78,144 \\
\hline Mean & 81989 & & 11712 & \\
\hline
\end{tabular}

Source: [28]

Table-IV shows, the trends of share of women student's enrollment and graduation in undergraduate program in terms of number from the total students which show increment but still low as compared to male students. Figure 4 also shows, share of women in post graduate program that indicates very low participation position.

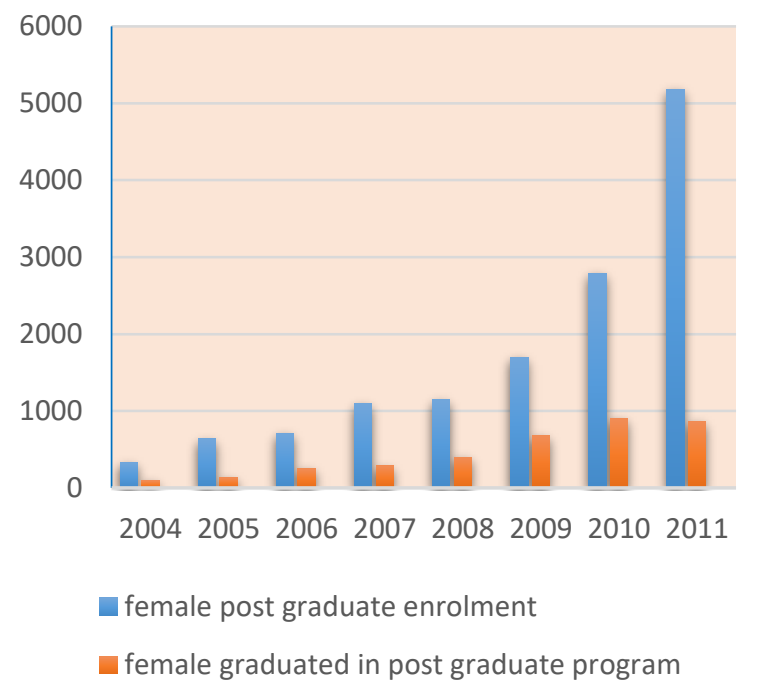

Fig. 4.The share of women students in post graduate program.
Table-V: Women Enrolled in Regular Undergraduate Engineering and Technology Education by Universities

\begin{tabular}{|c|c|c|c|c|c|c|}
\hline \multirow{2}{*}{$\begin{array}{c}\text { Univ } \\
\text { ersiti } \\
\text { es }\end{array}$} & \multirow{2}{*}{ Gender } & \multicolumn{5}{|c|}{ Year } \\
\hline & & 2007 & 2008 & 2009 & 2010 & 2011 \\
\hline $\begin{array}{l}\text { 䒰 } \\
\text { 宽 }\end{array}$ & $\begin{array}{c}\text { Male } \\
\text { Female } \\
\text { Total }\end{array}$ & $\begin{array}{c}1443 \\
379 \\
1822\end{array}$ & $\mathrm{Na}$ & $\begin{array}{c}4876 \\
865 \\
5741\end{array}$ & $\begin{array}{c}5736 \\
776 \\
6512\end{array}$ & $\begin{array}{c}5956 \\
969 \\
6925\end{array}$ \\
\hline 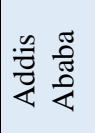 & $\begin{array}{c}\text { Male } \\
\text { Female } \\
\text { Total }\end{array}$ & $\begin{array}{c}1205 \\
408 \\
1613\end{array}$ & $\begin{array}{l}3164 \\
1097 \\
4261\end{array}$ & $\begin{array}{c}2753 \\
955 \\
3708\end{array}$ & $\begin{array}{c}2969 \\
910 \\
3879\end{array}$ & $\begin{array}{c}7836 \\
2799 \\
10635\end{array}$ \\
\hline 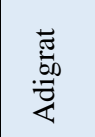 & $\begin{array}{c}\text { Male } \\
\text { Female } \\
\text { Total }\end{array}$ & $\mathrm{Na}$ & $\mathrm{Na}$ & $\mathrm{Na}$ & $\mathrm{Na}$ & $\begin{array}{l}173 \\
207 \\
380\end{array}$ \\
\hline 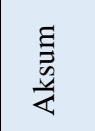 & $\begin{array}{c}\text { Male } \\
\text { Female } \\
\text { Total }\end{array}$ & $\begin{array}{c}38 \\
8 \\
46\end{array}$ & $\begin{array}{l}73 \\
13 \\
86\end{array}$ & $\begin{array}{c}226 \\
55 \\
281\end{array}$ & $\begin{array}{c}857 \\
362 \\
1219\end{array}$ & $\begin{array}{l}1794 \\
1027 \\
2821\end{array}$ \\
\hline 嵩 & $\begin{array}{l}\text { Male } \\
\text { Female } \\
\text { Total }\end{array}$ & $\mathrm{Na}$ & $\mathrm{Na}$ & $\begin{array}{c}281 \\
54 \\
335\end{array}$ & $\begin{array}{l}785 \\
148 \\
933\end{array}$ & $\begin{array}{l}785 \\
148 \\
933\end{array}$ \\
\hline 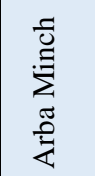 & $\begin{array}{c}\text { Male } \\
\text { Female } \\
\text { Total }\end{array}$ & $\begin{array}{c}1789 \\
346 \\
2135\end{array}$ & $\begin{array}{c}752 \\
276 \\
1028\end{array}$ & $\begin{array}{c}2931 \\
711 \\
3642\end{array}$ & $\begin{array}{l}5496 \\
1092 \\
6588\end{array}$ & $\begin{array}{l}6199 \\
1362 \\
7561\end{array}$ \\
\hline $\begin{array}{l}\tilde{n} \\
\tilde{W} \\
W \\
\hat{4}\end{array}$ & $\begin{array}{l}\text { Male } \\
\text { Female } \\
\text { Total }\end{array}$ & $\mathrm{Na}$ & $\mathrm{Na}$ & $\mathrm{Na}$ & $\mathrm{Na}$ & $\begin{array}{l}169 \\
205 \\
374\end{array}$ \\
\hline $\begin{array}{l}\text { คี } \\
\text { :ี } \\
\text { 苛 }\end{array}$ & $\begin{array}{c}\text { Male } \\
\text { Female } \\
\text { Total }\end{array}$ & $\begin{array}{c}2164 \\
452 \\
2616\end{array}$ & $\mathrm{Na}$ & $\begin{array}{c}2768 \\
749 \\
3517\end{array}$ & $\begin{array}{l}3548 \\
1087 \\
4635\end{array}$ & $\begin{array}{l}4469 \\
1358 \\
5827\end{array}$ \\
\hline 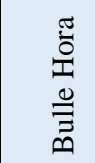 & $\begin{array}{c}\text { Male } \\
\text { Female } \\
\text { Total }\end{array}$ & $\mathrm{Na}$ & $\mathrm{Na}$ & $\mathrm{Na}$ & $\mathrm{Na}$ & $\begin{array}{l}58 \\
26 \\
84\end{array}$ \\
\hline 㤐壳 & $\begin{array}{c}\text { Male } \\
\text { Female } \\
\text { Total }\end{array}$ & $\mathrm{Na}$ & $\begin{array}{c}122 \\
45 \\
167\end{array}$ & $\begin{array}{c}44 \\
6 \\
50\end{array}$ & $\begin{array}{c}917 \\
411 \\
1328\end{array}$ & $\begin{array}{c}1503 \\
785 \\
2288\end{array}$ \\
\hline 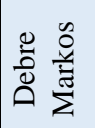 & $\begin{array}{l}\text { Male } \\
\text { Female } \\
\text { Total }\end{array}$ & $\mathrm{Na}$ & $\mathrm{Na}$ & $\begin{array}{c}368 \\
54 \\
420\end{array}$ & $\mathrm{Na}$ & $\begin{array}{c}2077 \\
6112 \\
688\end{array}$ \\
\hline
\end{tabular}




\begin{tabular}{|c|c|c|c|c|c|c|}
\hline 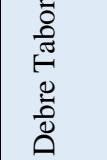 & $\begin{array}{c}\text { Male } \\
\text { Female } \\
\text { Total }\end{array}$ & $\mathrm{Na}$ & $\mathrm{Na}$ & $\mathrm{Na}$ & $\mathrm{Na}$ & $\begin{array}{c}99 \\
64 \\
163\end{array}$ \\
\hline$\stackrel{\widetilde{\sigma}}{\overline{\vec{\theta}}}$ & $\begin{array}{c}\text { Male } \\
\text { Female } \\
\text { Total }\end{array}$ & $\mathrm{Na}$ & $\mathrm{Na}$ & $\mathrm{Na}$ & $\mathrm{Na}$ & $\begin{array}{l}574 \\
329 \\
903\end{array}$ \\
\hline 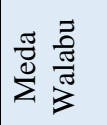 & $\begin{array}{c}\text { Male } \\
\text { Female } \\
\text { Total }\end{array}$ & $\mathrm{Na}$ & $\mathrm{Na}$ & $\begin{array}{l}38 \\
15 \\
53\end{array}$ & $\begin{array}{l}535 \\
170 \\
705\end{array}$ & $\begin{array}{c}1048 \\
3711 \\
419\end{array}$ \\
\hline 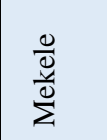 & $\begin{array}{c}\text { Male } \\
\text { Female } \\
\text { Total }\end{array}$ & $\begin{array}{c}1276 \\
268 \\
1544\end{array}$ & $\mathrm{Na}$ & $\begin{array}{c}1753 \\
293 \\
2046\end{array}$ & $\begin{array}{c}3497 \\
825 \\
4322\end{array}$ & $\begin{array}{c}36757 \\
904 \\
465\end{array}$ \\
\hline$\sum_{\sum}^{\mathbb{E}}$ & $\begin{array}{c}\text { Male } \\
\text { Female } \\
\text { Total }\end{array}$ & $\mathrm{Na}$ & $\mathrm{Na}$ & $\mathrm{Na}$ & $\mathrm{Na}$ & $\begin{array}{c}85 \\
41 \\
126\end{array}$ \\
\hline 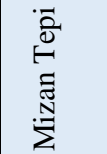 & $\begin{array}{c}\text { Male } \\
\text { Female } \\
\text { Total }\end{array}$ & $\mathrm{Na}$ & $\mathrm{Na}$ & $\begin{array}{c}482 \\
72 \\
554\end{array}$ & $\begin{array}{c}860 \\
287 \\
1147\end{array}$ & $\begin{array}{c}1486 \\
6992 \\
185\end{array}$ \\
\hline 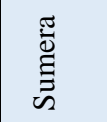 & $\begin{array}{c}\text { Male } \\
\text { Female } \\
\text { Total }\end{array}$ & $\mathrm{Na}$ & $\mathrm{Na}$ & $\begin{array}{c}29 \\
8 \\
37\end{array}$ & $\mathrm{Na}$ & $\begin{array}{l}109 \\
184 \\
293\end{array}$ \\
\hline 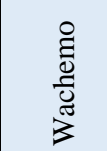 & $\begin{array}{c}\text { Male } \\
\text { Female } \\
\text { Total }\end{array}$ & $\mathrm{Na}$ & $\mathrm{Na}$ & $\mathrm{Na}$ & $\mathrm{Na}$ & $\begin{array}{c}114 \\
64 \\
178\end{array}$ \\
\hline $\begin{array}{l}\stackrel{0}{00} \\
\stackrel{0}{0} \\
3 \\
3\end{array}$ & $\begin{array}{c}\text { Male } \\
\text { Female } \\
\text { Total }\end{array}$ & $\begin{array}{c}125 \\
20 \\
145\end{array}$ & $\mathrm{Na}$ & $\begin{array}{c}332 \\
80 \\
472\end{array}$ & $\begin{array}{c}1093 \\
233 \\
1326\end{array}$ & $\begin{array}{c}2257 \\
7292 \\
986\end{array}$ \\
\hline $\begin{array}{l}\stackrel{0}{\overline{0}} \\
3\end{array}$ & $\begin{array}{c}\text { Male } \\
\text { Female } \\
\text { Total }\end{array}$ & $\mathrm{Na}$ & $\begin{array}{c}139 \\
25 \\
164\end{array}$ & $\begin{array}{c}1127 \\
158 \\
1285\end{array}$ & $\begin{array}{c}1572 \\
353 \\
1925\end{array}$ & $\begin{array}{c}1477 \\
2981 \\
775\end{array}$ \\
\hline 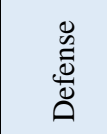 & $\begin{array}{c}\text { Male } \\
\text { Female } \\
\text { Total }\end{array}$ & $\mathrm{Na}$ & $\mathrm{Na}$ & $\begin{array}{c}531 \\
10 \\
541\end{array}$ & $\begin{array}{c}531 \\
10 \\
541\end{array}$ & $\begin{array}{c}531 \\
10 \\
541\end{array}$ \\
\hline $\begin{array}{l}\frac{\pi}{3} \\
0 \\
0 \\
0 \\
0\end{array}$ & $\begin{array}{c}\text { Male } \\
\text { Female } \\
\text { Total }\end{array}$ & $\mathrm{Na}$ & $\begin{array}{c}158 \\
37 \\
195\end{array}$ & $\begin{array}{c}806 \\
558 \\
1364\end{array}$ & $\begin{array}{c}1413 \\
686 \\
2099\end{array}$ & $\begin{array}{c}2313 \\
10323 \\
345\end{array}$ \\
\hline 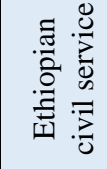 & $\begin{array}{c}\text { Male } \\
\text { Female } \\
\text { Total }\end{array}$ & $\begin{array}{c}328 \\
39 \\
367\end{array}$ & $\mathrm{Na}$ & $\begin{array}{c}562 \\
57 \\
619\end{array}$ & $\begin{array}{c}484 \\
44 \\
528\end{array}$ & $\begin{array}{c}474 \\
62 \\
536\end{array}$ \\
\hline
\end{tabular}

\begin{tabular}{|c|c|c|c|c|c|c|}
\hline $\begin{array}{l}\dot{\bar{v}} \\
\overline{0} \\
0\end{array}$ & $\begin{array}{c}\text { Male } \\
\text { Female } \\
\text { Total }\end{array}$ & $\mathrm{Na}$ & $\mathrm{Na}$ & $\mathrm{Na}$ & $\begin{array}{c}1054 \\
459 \\
1513\end{array}$ & $\begin{array}{c}2014 \\
972 \\
2986\end{array}$ \\
\hline 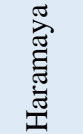 & $\begin{array}{c}\text { Male } \\
\text { Female } \\
\text { Total }\end{array}$ & $\begin{array}{l}531 \\
132 \\
663\end{array}$ & $\begin{array}{c}1254 \\
148 \\
1402\end{array}$ & $\begin{array}{c}1438 \\
205 \\
1643\end{array}$ & $\begin{array}{c}3506 \\
651 \\
4157\end{array}$ & $\begin{array}{c}4692 \\
861 \\
5553\end{array}$ \\
\hline 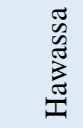 & $\begin{array}{c}\text { Male } \\
\text { Female } \\
\text { Total }\end{array}$ & $\begin{array}{c}838 \\
175 \\
1013\end{array}$ & $\begin{array}{c}1899 \\
368 \\
2267\end{array}$ & $\begin{array}{c}2214 \\
448 \\
2662\end{array}$ & $\begin{array}{c}3833 \\
673 \\
4506\end{array}$ & $\begin{array}{l}5070 \\
1161 \\
6231\end{array}$ \\
\hline 芯 & $\begin{array}{c}\text { Male } \\
\text { Female } \\
\text { Total }\end{array}$ & $\mathrm{Na}$ & $\mathrm{Na}$ & $\mathrm{Na}$ & $\begin{array}{c}851 \\
186 \\
1,037\end{array}$ & $\begin{array}{c}1406 \\
532 \\
1938\end{array}$ \\
\hline 䓂 & $\begin{array}{c}\text { Male } \\
\text { Female } \\
\text { Total }\end{array}$ & $\begin{array}{c}935 \\
167 \\
1102\end{array}$ & $\mathrm{Na}$ & $\begin{array}{c}1878 \\
294 \\
2171\end{array}$ & $\begin{array}{c}3727 \\
477 \\
4204\end{array}$ & $\begin{array}{c}6097 \\
860 \\
6957\end{array}$ \\
\hline $\begin{array}{l}\text { 莮 } \\
\frac{\pi}{0} \\
3 \\
3\end{array}$ & $\begin{array}{c}\text { Male } \\
\text { Female } \\
\text { Total }\end{array}$ & $\mathrm{Na}$ & $\mathrm{Na}$ & $\mathrm{Na}$ & $\begin{array}{l}694 \\
192 \\
886\end{array}$ & $\begin{array}{c}1665 \\
625 \\
2290\end{array}$ \\
\hline$\frac{\pi}{0}$ & $\begin{array}{c}\text { Male } \\
\text { Female } \\
\text { Total } \\
\end{array}$ & $\mathrm{Na}$ & $\mathrm{Na}$ & $\mathrm{Na}$ & $\mathrm{Na}$ & $\begin{array}{c}79 \\
84 \\
163\end{array}$ \\
\hline $\begin{array}{l}\frac{0}{4} \\
\frac{\pi}{0} \\
3\end{array}$ & $\begin{array}{c}\text { Male } \\
\text { Female } \\
\text { Total }\end{array}$ & $\mathrm{Na}$ & $\mathrm{Na}$ & $\mathrm{Na}$ & $\mathrm{Na}$ & $\begin{array}{l}209 \\
258 \\
467\end{array}$ \\
\hline
\end{tabular}

Source: [28], $\mathrm{Na}=$ data not available

Table-v show, the share of female student's enrolment in regular undergraduate program in engineering and technology education in 31 public universities from the year 2007-2011 which shows there is grate disparity and under representation of female engineering education participation even if there is some improvement. The pattern of engineering enrolment is various in each universities. Since some of the universities are new established recently there is no data give for them. Especially third generation universities started education 2003/2004 data is not available for them. Hence, Addis Ababa University, Adama University, Aksum university, Mekele University, Haramaya, Hawassa, Jimma, Arba Minch, Bahir Dar, Wollega and Defiance Universes are the old universities and the most Engineering and technology education provider. The summation of Engineering student in this universities are summarized in the following table 6 and figure 4 including enrolment in non- government university and collages.

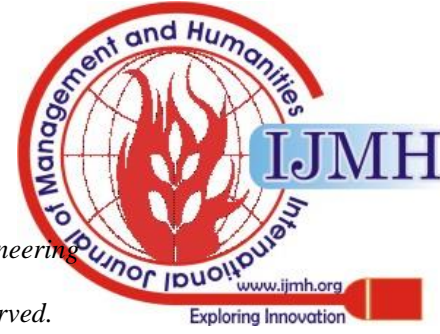




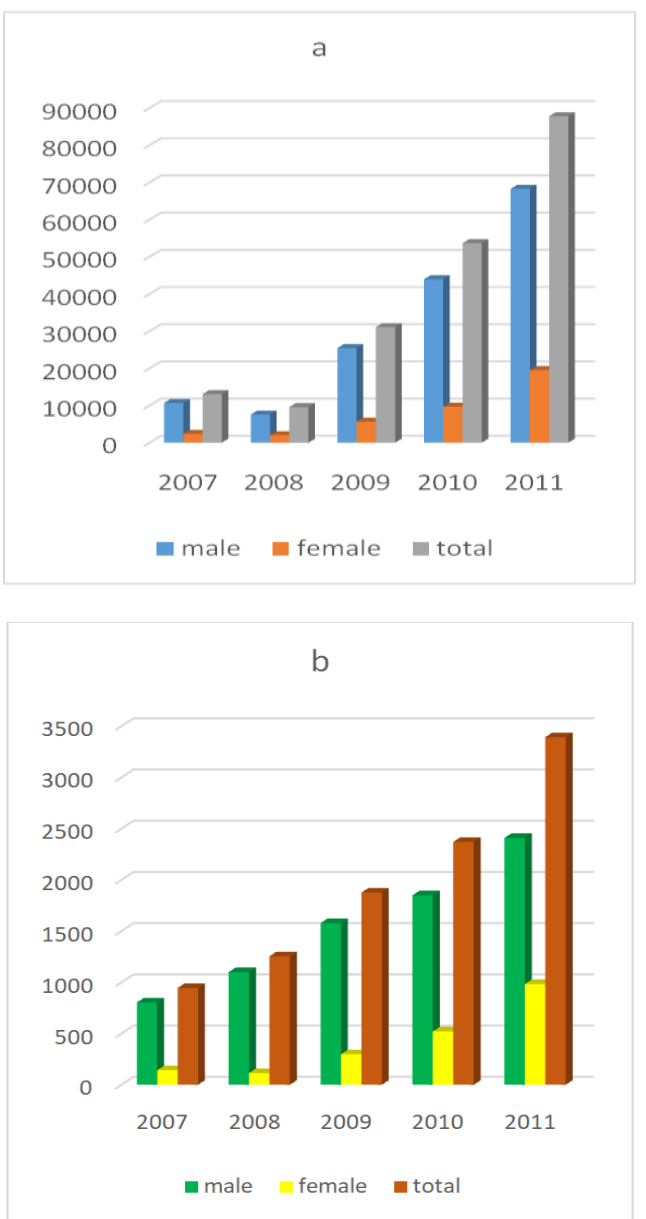

Fig. 5 a and $b$. trends and share of women regular undergraduate enrolment in engineering and technology education at government and non-government universities respectively.

In figure $5 \mathrm{a}$ and $\mathrm{b}$, on the bases of data [28], the share of women enrolment in engineering and technology education participation is increased from 18\% during 2007 to $22.23 \%$ during 2011 in government universities and from 15\% to $28 \%$ in non-government universities and colleges. However, there is gender disparity and women are underrepresented in engineering and technology education.

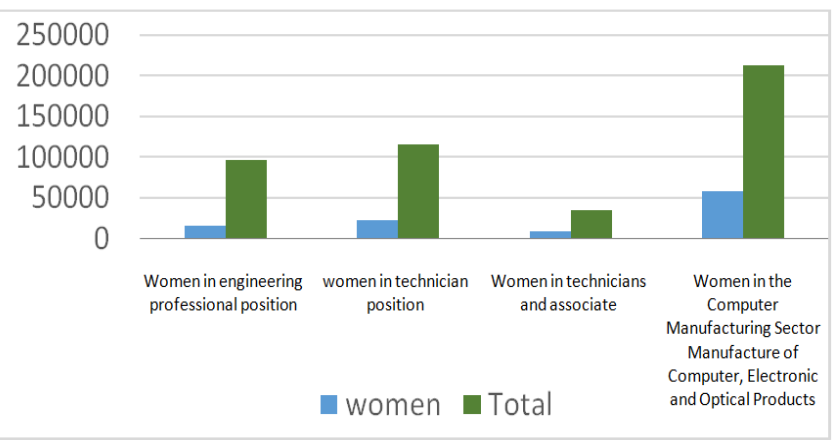

Fig. 6. Share of women from the total employments of engineering and technology fields

Figure 6 is constructed on the bases of data from national assessment [10] shows, the share of women in engineering and technology employment such as, technician position, engineering science, technicians and associate technician, computer manufacturing and others areas according to available data at national assessment 2013. The data shows that the share of women employees in this profession is very low but they are better in manufacturing employment.

\section{CONCLUSION}

The finding of this study indicate that, women are underrepresented and there is grate disparities between male and female participation regarding to engineering and technology enrollment, graduation, employment and profession position in the field which is in line with the literature reviewed. This implies that problem needs attention so that give notice for the problem as well as creating awareness about the importance of engineering education starting from lower class through encouraging girls in science subjects and also take experience from other countries. In addition creating suitable environment for engineers at university level is necessary and there should be future research investigation in the field to identify the reason behind underrepresentation of women in engineering education and employment which can suggest sustainable solution for this problem.

\section{REFERENCES}

1. Abebayehu Tora (2013) Assessment of Sexual Violence against Female Students in Wolaita Sodo University, Southern Ethiopia, Journal of Intertamporal violence vol.8, issue 11

2. J. Gill et al. (2008) I still want to be an engineer! Women, education and the engineering profession, European Journal of Education, 33:4 391-402, DOI: 10.1080/03043790802253459

3. Addissie .M and Singh. S (2018), Experience of women in engineering education: literature review, Asia Pacific Journal of Research, Vol: I. Issue LVV, pp174-178

4. Dimitriadi (2013) Young women in science and technology: the importance of choice, Journal of Innovation and Entrepreneurship vol. 2:No.5pp1-14:http://www.innovation-entrepreneurship.com/content/2 $/ 1 / 5$

5. Robsan Margo Egne (2014) Gender Equality in Public Higher Education Institutions of Ethiopia: The Case of Science, Technology, Engineering, and Mathematics, Discourse and Communication for Sustainable Education, vol. 5, pp. 3ñ21, 2014: DOI: 10.2478/dcse20140001

6. Tamiru Jote (2017), Exploring Employment Status and Education-Job Match among Engineering Graduates in Ethiopia: Policy Implications

7. Samira I. Islam (2017) Arab Women in Science, Technology, Engineering and Mathematics Fields: The Way Forward, World Journal of Education Vol. 7, No. 6,pp 12-20, doi:10.5430/wje.v7n6p12, ISSN 1925-0746 E-ISSN 1925-0754

8. Choudhury (2015) explaining gender discrimination in the employment and earnings of engineering graduate's in India. Journal of educational planning and administration, vol.XXIX, No.3, pp 225-246

9. Addissie .M and Singh. S (2018), factors affecting academic performance of women in engineering education and their experience of participation in employment: literature review, international Journal of Research, Volume 05 Issue 01 pp 3733-3739

10. Helina Beyene (2015) final report national assessment: Ethiopia gender equality and the knowledge society, women in global science and technology,

11. World bank country study(2005), education in Ethiopia strengthening the foundation for sustainable progress, ISBN-10: 0-8213-6226-7 ISBN-13: 978-0-8213-6226-6, ISSN: 0253-2123 DOI: 10.1596/978-0-8213-6226-6

12. Ethiopia socioeconomic survey(2015/2016) report by A Report by the Central Statistical Agency of Ethiopia in Collaboration with the National Bank of Ethiopia and the World Bank, February 2017

13. A. Powell et al. (2011) A poisoned chalice? Why UK women engineering and technology students may receive more 'help' than their male peers, Gender and Education, 23:5, 585- 599, DOI:

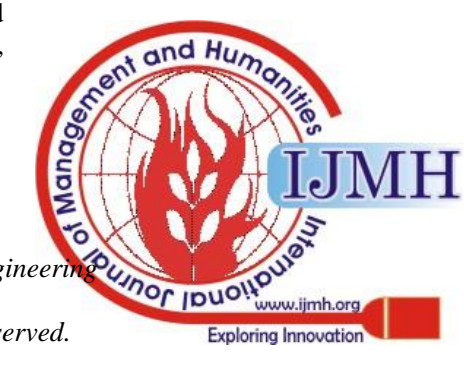


$10.1080 / 09540253.2010 .527826$

14. Alice E. Smith \& Berna Dengiz (2010) Women in engineering in Turkey - a large scale quantitative and qualitative examination, European Journal of Engineering Education, 35:1, 45-57, DOI: $10.1080 / 03043790903406345$

15. Catherine Hill et al. (2010) Women in Science, Technology, and Engineering, and Mathematics, page xiv, Library of Congress Control Number: 2010901076 ISBN: 978-1-879922-40- 2 Published by AAUW Washington, DC 20036 Web: www.aauw.org

16. Carmen García Villa \& Elsa M. González y González (2014) Women students in engineering in Mexico: exploring responses to gender differences, International Journal of Qualitative Studies in Education, 27:8, 1044-1061, DOI: 10.1080/09518398.2014.924636

17. C. Riegle-Crumb, C. Moore (2013) Examining Gender Inequality In A High School Engineering Course, American Journal of Engineering Education Special Edition Volume 4, Number 1 pp55- 66, http://creativecommons.org/licenses/by/3.0/

18. Elsa Q. Villa et al.(2016), Engineering Education through the Latina Lens, Engineering Education through the Latina Lens, Journal of Education and Learning; Vol. 5, No. 4; ISSN 19275250 E- ISSN 1927-5269, doi:10.5539/jel.v5n4p113

19. Ghiasi G, Larivière V, Sugimoto CR (2015) On the Compliance of Women Engineers with a Gendered Scientific System. PLoS ONE vol.10, No.12, pp1-19: e0145931. doi:10.1371/journal.pone.0145931

20. Kacey D. Beddoes (2012) Feminist Scholarship in Engineering Education: Challenges and Tensions, Engineering Studies, 4:3, 205-232, DOI:10.1080/19378629.2012.693932

21. Maria Udeân (2002) the Impact of Women on Engineering: A Study of Female Engineering Students' Thesis Topics, International Journal of Engineering Education. Vol. 18, No. 4, pp. 458, 2002 0949-149X/91 $\$ 3.00+0.00$ Printed in Great Britain at TEMPUS Publications

22. Masako Hosaka (2014) Women's experiences in the engineering laboratory in Japan, European Journal of Engineering Education, 39:4 424-431, DOI: 10.1080/03043797.2014.883363

23. S. Barnard, T. Hassan, B. Bagilhole \& A. Dainty (2012) 'They're not girly girls': an exploration of quantitative and qualitative data on engineering and gender in higher education, European Journal of Engineering Education, vol.37:No.2, pp193-204, DOI: $10.1080 / 03043797.2012 .661702$

24. Singh. S, S. Fenton (2014) Women Engineers: A Comparative Study between India and Australia International Journal of Advancements in Research \& Technology, Volume 3, Issue 7, pp108-121, ISSN 2278-7763

25. Jean Bossart, Neelam Bharti (2017) Women in Engineering: Insight into Why Some Engineering Departments Have More Success in Recruiting and Graduating Women, American Journal of

Engineering Education Volume 8, Number 2 pp127-140http://creativecommons.org/licenses/by/3.0/

26. Singh.S (2013) Women in Engineering Education in India http://www.youblisher.com/p/109258-

Women-in-Engineering-Education-in-India/

27. https://en.wikipedia.org/wiki/Education in Ethiopia)

28. Ethiopian ministry of education educational annual abstract 2000/01-2012, Addis Ababa Ethiopia.

29. World Development Indicators database (2018).

30. Development Indicators of Amhara Region (2011/12), Bureau of Finance and Economic Development, pp80

31. MOFED (2002) ministry of finance and Economic Development, Addis Ababa, Ethiopia pp.

32. UNISCO (2018)

Statistics,http://uis.unesco.org/country/ET

Institutes

of

\section{AUTHORS PROFILE}

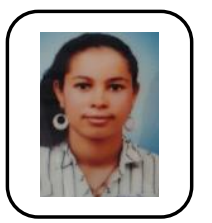

Addissie Melak, Department of Humanities Delhi Technological University, Delhi, India, ${ }^{\text {b }}$ Department of Economics, Debre Tabor University, Ethiopia.

Ph.D Research Scholar in Economics, Department of Humanities Delhi Technological University, Delhi, India, M.A in Economics at Punjabi University, Patiala, India, Bachelor Art Degree in Economics at Debre Markos University, Ethiopia, , Working staff in Department of Economics at Debre Tabor University, Ethiopia. Four international conferences presentation Participation. Four paper published in the Journals. addmelak24@gmail.com

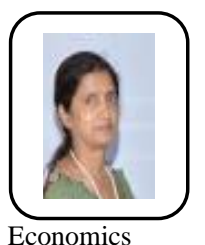

Professor Seema Singh, Department of Humanities Delhi Technological University, Delhi, India, INWES Board Member (South Asia) \& Vice President (Education \& Research)

International Network of Women Engineers and Scientists (INWES)

Hon. Joint Secretary Indian Society of Labour

Hon. Vice President, Women in Science \& Engineering \&

Hon. Vice President -I, University Women Association of Delhi.

X - HOD of Department of Humanities. More than 15 international conference participation, more than 40 articles published, wrote four book, advisor of more than 10 students.....seemahumanitiesdtu@gmail.com 